\title{
Adsorption Behaviors of Straight-Chain Alkanes on a Molybdenite [001]/[100] Surface: A Molecular Dynamics Study
}

\author{
He Wan ${ }^{1, * \mathbb{D}}$, Peng $\mathrm{Yi}^{1}{ }^{1}$, Juanping $\mathrm{Qu}{ }^{1,2}$, Xianzhong Bu ${ }^{1, *}$, Wei Yang ${ }^{1}$ and $\mathrm{Hui} \mathrm{Li}^{3}$ \\ 1 School of Resources Engineering, Xi'an University of Architecture and Technology, Xi'an 710055, China; \\ yipeng@xauat.edu.cn (P.Y.); qjp@live.xauat.edu.cn (J.Q.); yangwei@xauat.edu.cn (W.Y.) \\ 2 State Environmental Protection Key Laboratory of Mineral Metallurgical Resources Utilization and Pollution \\ Control, Wuhan University of Science and Technology, Wuhan 430081, China \\ 3 College of Resource and Civil Engineering, Northeastern University, Shenyang 110819, China; \\ lihui_cl@xauat.edu.cn \\ * Correspondence: wanhe@xauat.edu.cn (H.W.); buxianzhong@xauat.edu.cn (X.B.); \\ Tel.: +86-029-8220-3408 (H.W. \& X.B.)
}

check for updates

Citation: Wan, H.; Yi, P.; Qu, J.; Bu,

X.; Yang, W.; Li, H. Adsorption

Behaviors of Straight-Chain Alkanes on a Molybdenite [001]/[100] Surface: A Molecular Dynamics Study. Minerals 2021, 11, 489. https:// doi.org/10.3390/min11050489

Academic Editor: William Skinner

Received: 20 March 2021

Accepted: 2 May 2021

Published: 4 May 2021

Publisher's Note: MDPI stays neutral with regard to jurisdictional claims in published maps and institutional affiliations.

Copyright: (c) 2021 by the authors. Licensee MDPI, Basel, Switzerland. This article is an open access article distributed under the terms and conditions of the Creative Commons Attribution (CC BY) license (https:// creativecommons.org/licenses/by/ $4.0 /)$.

\begin{abstract}
Straight-chain alkanes (SCA) as collectors can effectively enhance the floatability of molybdenite. In a previous study, SCA were found to have an excellent adsorption effect on the molybdenite [001] surface (MS001), but they exhibited no adsorption behavior on the molybdenite [100] surface (MS100). However, other studies have shown that SCA could adsorb on MS100. In this paper, the underlying cause of this contradictory conclusion was identified by molecular dynamics simulation. The results show that SCA could adsorb both MS001 and MS100. However, at low SCA dosages, SCA have a strong interaction with MS001 but desorb quickly on MS100. This leads to the selective adsorption of SCA on MS001. As SCA's concentration gradually increases, the selective adsorption behavior of SCA on MS001 will be disrupted. Excessive SCA concentration will lead to its adsorption at MS100.
\end{abstract}

Keywords: molecular dynamics; adsorption behaviors; molybdenite surface; straight-chain alkanes

\section{Introduction}

Straight-chain alkanes (SCA) are a superior collector of molybdenite [1-4]. They can enhance the floatability of molybdenite by adsorbing on the surface of molybdenite $[4,5]$. Some studies have reported that SCA could only adsorb on the surface of molybdenite (001) (MS001) by Van der Waals force, but not on the surface of molybdenite (100) (MS100) [6-8] based on the anisotropy of molybdenite $[9,10]$. It has also been agreed that as the molybdenite's particle size gradually decreases, the proportion of MS100 increases [11,12]. This causes the SCA's effect of enhancing the floatability of molybdenite to become less effective, and the recovery of molybdenite flotation also decreases.

However, it has also been reported that when the amount of SCA increases, the rate of flotation recovery of fine molybdenite gradually increases $[13,14]$. This indicates that SCA may be adsorbed not only on the MS001 but also on the MS100. This conclusion is contrary to the findings of previous reports. The reason why SCA can adsorb on the MS100 is not yet clear.

In this paper, molecular dynamics simulations were used to obtain the lowest energy conformation of SCA on MS001/MS100, calculate the interaction energy of SCA and MS001/MS100, and analyze the dynamics behavior of SCA on MS001/MS100. The reason why SCA can adsorb on MS100 is explained at the molecular level.

\section{Molecular Dynamics Simulation}

Models of the surface of molybdenite and SCA (dodecane representing SCA) were established on the Materials Visualizer module and Amorphous Cell module. Molecular 
dynamics simulations were performed under the Universal force field in the Forcite Plus module with the software package Materials Studio 8.0 (Northeastern University, Shenyang, Liaoning, China).

\subsection{Simulated Detail}

Firstly, molybdenite crystal (MS001/MS100) was established and optimized based on the method published in the literature [15] (Figure 1).

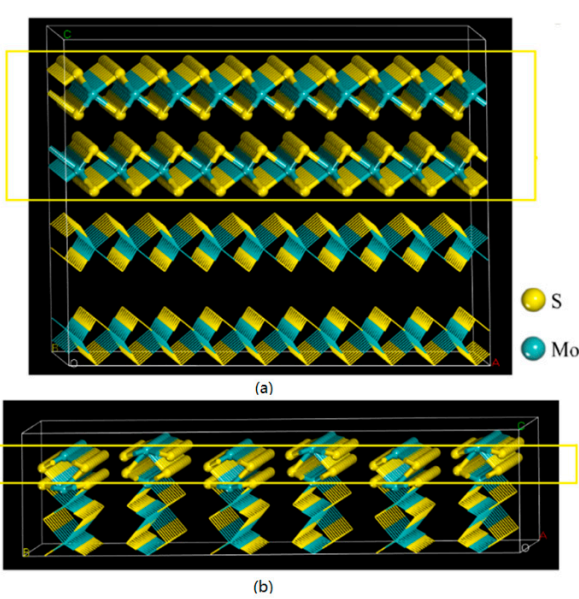

Figure 1. The optimization of MS001/MS100. (a) MS001; (b) MS100.

Secondly, MS001/MS100 were cut at depths of 2 and $4 \AA$, respectively. They were then extended to $3 \times 10$ - and $10 \times 10$-unit supercells, respectively, and a vacuum layer of $0 \AA$ was established for combination with SCA boxes.

Thirdly, an MS001/MS100-SCA complex model was optimized to obtain the optimum position in the adsorption equilibrium by using the same method as the optimization of molybdenite crystal (Figure 2). A cutoff distance for group-based summation was $15.5 \AA$. The atomic charge was calculated using the QEq charge equilibration method [16].

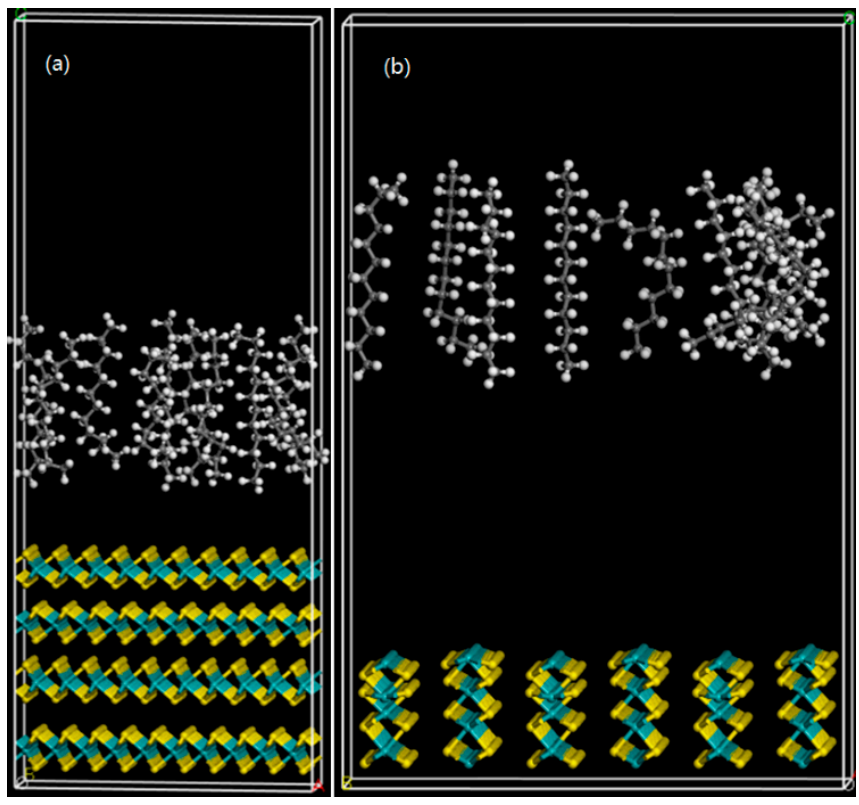

Figure 2. The optimization of the MS001/MS100-SCA compound model. (a) SCA and MS001; (b) SCA and MS100. 
Finally, molecular dynamics simulations were carried out as follows [15]: (1) The configuration's initial velocity was obtained by the Velocity Scale thermostat of the NVT ensemble. (2) At a time step of $1 \mathrm{fs}$ and a total simulation time of $50 \mathrm{ps}$, the NVE ensemble carried out annealing molecular dynamics simulations. (3) Molecular dynamics simulations with the NVT ensemble were performed using a Nosé thermostat with a $Q$ ratio of 0.01 , at $293 \mathrm{~K}$, a time step of $1 \mathrm{fs}$, and a total simulation time of $3000 \mathrm{ps}$.

\subsection{Interaction Energy Calculation}

The interaction energy was calculated based on the configuration with the lowest energy - the optimal adsorption model. The interaction energy was obtained by the following formula [17]:

$$
\Delta \mathrm{E}=\mathrm{E}_{\mathrm{total}}-\left(\mathrm{E}_{\mathrm{MS} 001 / \mathrm{MS} 100}+\mathrm{E}_{\mathrm{SCA}}\right)
$$

where $\Delta \mathrm{E}$ is the interaction energy; $\mathrm{E}_{\text {total }}, \mathrm{E}_{\mathrm{MS} 001 / \mathrm{MS} 100}$, and $\mathrm{E}_{\mathrm{SCA}}$ are the total energy of the complex model, MS001/MS100, and SCA, respectively.

\subsection{Square Displacement (MSD) Curves}

MSD curves were used to analyze the aggregation properties of SCA on the MS001/MS100. In the MSD curves, the mobility of SCA is affected by the properties of MS001/MS100. The relationship between MSD and the diffusion coefficients (D) is based on the related literature, as follows [18,19]:

$$
D=\lim _{t \rightarrow \infty}\left(\frac{M S D}{6 t}\right)=\frac{1}{6} K_{M S D}
$$

where $K_{M S D}$ is the slope of the MSD curve.

\section{Results and Discussion}

\subsection{The Adsorption Configuration of SCA on the MS001/MS100}

The adsorption configuration of SCA on the MS001/MS100 is shown in Figure 3.

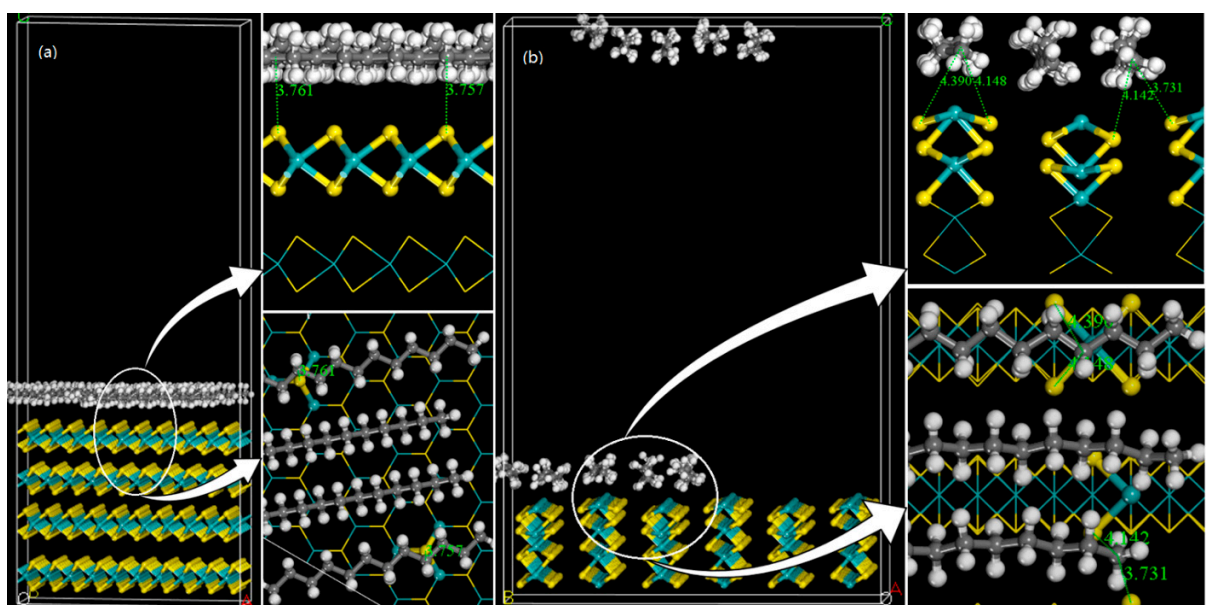

Figure 3. The adsorption behavior of SCA on the surface of molybdenite. (a) SCA and MS001; (b) SCA and MS100.

The results of Figure 3a show that all SCA adsorb in parallel on the MS001, which consists of sulfur atomic planes. Figure $3 b$ shows that SCA also adsorb on the MS100 composed of alternating sulfur and molybdenum atomic planes. They are arranged in parallel between sulfur atoms and sulfur atoms exposed on the MS100 (containing S-plane). Meanwhile, there is no SCA adsorption above the exposed Mo atom of the Mo plane. This suggests that the exposed sulfur atoms on the MS100 are beneficial to SCA's adsorption, while the exposed Mo atoms can hinder the spreading of SCA. 
This conclusion can be further explained by relying on the average partial charge of the atomic surface (Table 1).

Table 1. The averaged partial charges of SCA/MS001/MS100.

\begin{tabular}{cccc}
\hline Mineral & Crystal Surface & Surface Atoms & Averaged Partial Charges (eV) \\
\hline \multirow{3}{*}{ Molybdenite } & \multirow{2}{*}{100} & $\mathrm{~S}$ & -0.367 \\
\cline { 2 - 4 } & & Mo & +0.734 \\
\cline { 2 - 4 } & 001 & $\mathrm{~S}$ & -0.396 \\
\hline \multicolumn{2}{c}{ SCA } & $\mathrm{H}$ & +0.053 \\
\hline
\end{tabular}

The results of Table 1 show that the averaged partial charges of the surface S/Mo atoms in MS100 are $-0.367 /+0.734 \mathrm{eV}$. The averaged partial charges of the surface $\mathrm{H}$ atoms in SCA are $+0.053 \mathrm{eV}$. The surface $\mathrm{H}$ atoms of SCA and surface S atoms of MS001/MS100 can interact by electrostatic gravitation. The surface $\mathrm{H}$ atoms of SCA and surface Mo atoms of MS100 will repel each other. Therefore, the SCA can be adsorbed on the exposed sulfur atoms on the MS100, not on the surface Mo atoms of MS100. Even the surface Mo atoms of MS100 will block the adsorption of SCA.

\subsection{The Interaction Energy between SCA and the MS001/MS100}

To further analyze SCA's adsorption behavior on the surface of molybdenite, SCA's interaction energies with the MS001/MS100 were calculated. The value of interaction energy shows the adsorption system's stability (SCA's interaction strength with the MS001/MS100) [14]. A more negative interaction energy value indicates a more stable adsorption system (favorable interaction). The results are shown in Table 2.

Table 2. The interaction energy of SCA with the MS001/MS100.

\begin{tabular}{|c|c|c|c|c|}
\hline Mineral & Crystal Surface & Reagent & 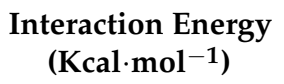 & $\begin{array}{l}\text { Standard } \\
\text { Deviation }\end{array}$ \\
\hline \multirow{2}{*}{ Molybdenite } & 100 & \multirow{2}{*}{ SCA } & -239.6827 & 10.6774 \\
\hline & 001 & & -559.5798 & 7.3797 \\
\hline
\end{tabular}

The results of Table 2 show that the interaction energy of SCA with the MS001/MS100 is $-559.5798 /-239.6827 \mathrm{Kcal} \cdot \mathrm{mol}^{-1}$. The interaction energy between SCA and MS001 is a much more negative value than that of SCA and MS100. Combining the results in Figure 1 shows that SCA's interaction with sulfur atoms on the MS001 is much stronger than its interaction with sulfur atoms on the MS100. Therefore, this can also cause SCA's preferential adsorption on MS001 when there is a low amount of SCA present. This is in agreement with the results of the averaged partial charges of SCA/MS001/MS100.

The results of Table 1 show that the averaged partial charges of the surface $S$ atoms in MS001 are $-0.396 \mathrm{eV}$. The surface H atoms of SCA and surface S atoms of MS001 can also interact by electrostatic gravitation. The interaction force between the surface $\mathrm{H}$ atoms of SCA and surface $S$ atoms of MS001 is greater than the interaction force between the surface $\mathrm{H}$ atoms of SCA and surface S atoms of MS100. Meanwhile, the repulsive force of MS100 to SCA is stronger than the attraction of MS100 to SCA. Therefore, the MS100 has a repulsive effect on SCA as a whole. However, the MS001 only has an attractive effect on SCA. This further indicates that SCA can be preferentially adsorbed on the MS001.

\subsection{The Diffusion Coefficients of SCA on the MS001/MS100}

MSD curves were used to analyze the stability of SCA on the MS001/MS100. MSD curves of SCA on MS001/MS100 are shown in Figure 4. 


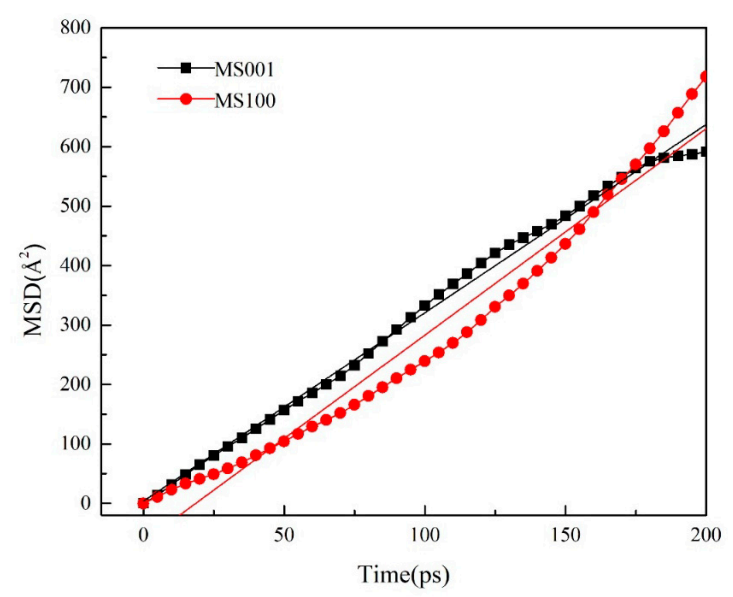

Figure 4. MSD curves of SCA on the MS001/MS100.

Figure 4 shows that SCA's diffusion coefficients are $3.17 \times 10^{-5} \mathrm{~cm}^{2} / \mathrm{s}$ and $3.47 \times 10^{-5} \mathrm{~cm}^{2} / \mathrm{s}$ on MS001 and MS100, respectively. These indicate that the mobility of SCA on the MS001 is slower than that of SCA on the MS100. This is attributed to the fact that the MS001 has a stronger attraction, which limits SCA movement more than it does on the MS100. Therefore, compared with the MS100, it is harder on MS001 to desorb SCA. This will cause more hydrocarbon oil to be retained on the MS001 under the same conditions.

\subsection{The Relative Concentration of SCA on the MS001/MS100}

To further analyze SCA's distribution on the MS001/MS100, the relative concentration of SCA on the MS001/MS100 is shown in Figure 5.

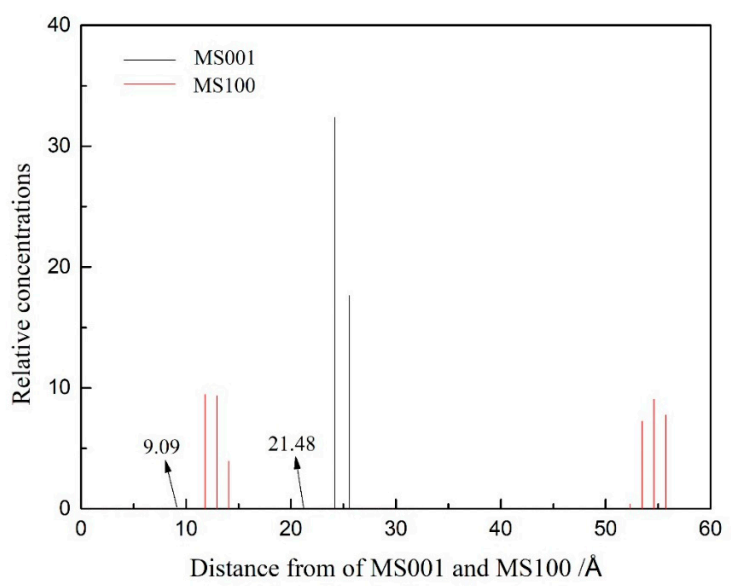

Figure 5. The relative concentration of SCA on the MS001/MS100. The distance of 21.48 $/ 9.09 \AA$ are the locations of the MS001/MS100, respectively.

Figure 5 shows that all SCA adsorbed on the MS001 were distributed between $24.19 \AA$ and $25.57 \AA$. The distance of SCA from the MS001 is approximately 2.71-4.09 $\AA$. The only portion of SCA that is adsorbed on the MS100 is distributed between $11.82 \AA$ and 14.07 $\AA$. The distance of SCA from the MS100 is approximately 2.73-4.98 $\AA$. At a distance of 2.7-5.0 ̊ from the MS001/MS100, SCA's adsorption on the MS001 is much higher than that of the SCA on the MS100. This is consistent with the findings in Figure 4, which indicate that adsorption of SCA is more favorable on the MS001.

\section{Discussion}

The results of the molecular dynamics simulations explain the reasons for the previous literature's contradictory conclusions. SCA can adsorb on the MS100, despite the low 
interactions between SCA and the MS100, the easy desorption of SCA on the MS100, and the blocking effect of the exposed molybdenum atoms of the MS100 on hydrocarbon oil. However, when the amount of SCA is low, it can selectively adsorb on the MS001, based on the blocking and strong desorption role of the MS100. At low SCA dosage, SCA can selectively adsorb on the MS001 but not on the MS100, based on the blocking, strong desorption, and weak interaction forces between SCA and the MS100. With the increasing SCA concentration, an excessive amount of SCA will reduce SCA's selectivity for adsorption on the MS001. This will cause a small amount of the SCA to be adsorbed on the MS100. This conclusion is consistent with results concerning oil agglomeration and the flotation of molybdenite [13,14].

\section{Conclusions}

Molecular dynamics simulations of SCA's adsorption behaviors on the surface of molybdenite found that SCA can be adsorbed on both MS001 and MS100. At a low SCA dosage, SCA can selectively adsorb on the MS001 but not on the MS100, based on the strong interactions between SCA and the MS001, the easy desorption of SCA on the MS100, and the blocking effect of the exposed molybdenum atoms of the MS100 on SCA. With an increasing SCA concentration, an excessive amount of SCA destroys SCA's selectivity for adsorption on the MS001. This will cause the adsorption of SCA on the MS100 to gradually increase.

Author Contributions: The manuscript was written through contributions of all authors. Conceptualization, H.W. and X.B.; methodology, P.Y.; software, H.L.; validation, P.Y. and J.Q.; formal analysis, H.W. and P.Y.; investigation, P.Y.; resources, W.Y.; data curation, P.Y.; writing—original draft preparation, P.Y.; writing — review and editing, H.W.; visualization, P.Y.; supervision, H.L.; project administration, X.B.; funding acquisition, H.W., X.B. and H.L. All authors have read and agreed to the published version of the manuscript.

Funding: This research was funded by the Open Foundation of the State Environmental Protection Key Laboratory of Mineral Metallurgical Resources Utilization and Pollution Control (Grant No. HB201906), the National Natural Science Foundation of China (Grant No. 52074206 and 51904222), and the Shaanxi International Cooperation and Exchange Project (Grant No. 2021KWZ-16).

Data Availability Statement: The data presented are available in the article.

Acknowledgments: Wuhan University of Science and Technology contributed funding; Northeastern University contributed Materials Studio 8.0.

Conflicts of Interest: The authors declare no conflict of interest.

\section{References}

1. Crozier, R.D. Flotation reagent practice in primary and by-product molybdenite recovery. Min. Mag. 1979, 140, 174-178.

2. Smit, F.J.; Bhasin, A.K. Relationship of petroleum hydrocarbon characteristics and molybdenite flotation. Int. J. Miner. Process. 1985, 15, 19-40.

3. He, T.S.; Wan, H.; Song, N.P.; Guo, L. The influence of composition of nonpolar oil on flotation of molybdenite. Miner. Eng. 2011, 24, 1513-1516. [CrossRef]

4. Kelebek, S. Critical surface tension of wetting and of floatability of molybdenite and sulfur. J. Colloid Interface Sci. 1988, 124, 504-514. [CrossRef]

5. Castro, S.; Lopez-Valdivieso, A.; Laskowski, J.S. Review of the flotation of molybdenite. Part I: Surface properties and floatability. Int. J. Miner. Process. 2016, 148, 48-58. [CrossRef]

6. Kainuma, Y.; Uyeda, R. On the Structure of Adsorbed Organic Long-Chain Molecules on the Cleavage Surface of Molybdenite. J. Phys. Soc. Jpn. 1950, 5, 199-200. [CrossRef]

7. Groszek, A.J. Preferential adsorption of long-chain normal paraffins on MoS2, WS2 and graphite from n-heptane. Nature 1964, 104, 680. [CrossRef]

8. Zanin, M.; Ametov, I.; Grano, S.; Zhou, L.; Skinner, W. A study of mechanisms affecting molybdenite recovery in a bulk copper/molybdenum flotation circuit. Int. J. Miner. Process. 2009, 93, 256-266. [CrossRef]

9. Krishnaswamy, P. Kinetics of the Aqueous Oxidation of Molybdenite and the Role of Crystal Anisotropy on the Electrochemical Mechanisms of the Process. Ph.D. Dissertation, University of California, Berkeley, CA, USA, 1981; pp. 11-12. 
10. Zhou, L. Molybdenite Flotation. Master's Thesis, Ian Wark Research Institute and University of South Australia, Mawson Lakes, Australia, May 2010; p. 14.

11. Chander, S.; Fuerstenau, D.W. On the natural floatability of molybdenite. Trans. Am. Inst. Min. Metall. Eng. 1972, 252, 62-69.

12. Yang, B.Q.; Song, S.X.; Lopez-Valdivieso, A. Effect of Particle Size on the Contact Angle of Molybdenite Powders. Miner. Process. Extr. Metall. Rev. 2014, 35, 208-215. [CrossRef]

13. Song, S.; Zhang, X.; Yang, B.; Lopez-Mendoza, A. Flotation of molybdenite fines as hydrophobic agglomerate. Sep. Purif. Technol. 2012, 98, 451-455. [CrossRef]

14. Fu, J.; Chen, K.; Hui, W. Recovering molybdenite from ultrafine waste tailings by oil agglomerate flotation. Miner. Eng. 2012, 39, 133-139.

15. Yang, Q.; Sharp, K.A. Atomic Charge Parameters for the Finite Difference Poisson Boltzmann Method Using Electronegativity Neutralization. J. Chem. Theory Comput. 2006, 2, 1152-1167. [CrossRef] [PubMed]

16. Yin, Y.; Pui, S.C.; Reginald, B.H.T. Molecular simulation study of the effect of various additives on salbutamol sulfate crystal habit. Mol. Pharmaceut. 2011, 8, 1910-1918.

17. Hao, H.; Li, L.; Yuan, Z.; Liu, J. Molecular arrangement of starch, Ca2+ and oleate ions in the siderite-hematite-quartz flotation system. J. Mol. Liq. 2018, 254, 349-356.

18. Snehasis, C.; Amalendu, C. Molecular dynamics simulations of aqueous $\mathrm{NaCl}$ and $\mathrm{KCl}$ solutions: Effects of ion concentration on the single-particle, pair, and collective dynamical properties of ions and water molecules. J. Chem. Phys. 2001, 115, 3732-3741.

19. Li, E.; Du, Z.; Yuan, S.; Cheng, F. Low temperature molecular dynamic simulation of water structure at sylvite crystal surface in saturated solution. Miner. Eng. 2015, 83, 53-58. [CrossRef] 